\title{
Performance Analysis of Several Functional Splits in C-RAN
}

\author{
Jialong Duan*, Xavier Lagrange* and Frédéric Guilloud ${ }^{\dagger}$ \\ *Networks, Security and Multimedia Department \\ †Signal and Communications Department \\ Télécom Bretagne \\ Email: firstname.lastname@telecom-bretagne.eu
}

\begin{abstract}
Centralized/Cloud Radio Access Network (C-RAN) is a promising future mobile network architecture, which can potentially increase the capacity of mobile network meanwhile reducing operators' cost. In standard C-RAN, frequency shifting is made in Remote Radio Heads (RRHs), which are close to the antennas. Signal processing and upper layers are made in Baseband Unit (BBU) pool for multiple base stations. However, this results in high burden on the optical transport network between RRHs and BBU pool. This paper investigates new functional split architectures between RRH and BBU, to reduce the transmission throughput between RRHs and BBUs. Two new architectures are proposed and modeled for the uplink. We propose to move part of physical layer functions of the BBU to the RRH. For the proposed architectures, the transmission rate between RRHs and BBUs depends on the mobile network load, while that of current architecture is constant. Simulation results illustrate that $30 \%$ to $40 \%$ bandwidth can be saved when all the radio channel capacity is used, and up to $70 \%$ bandwidth when half of the radio channel capacity is used.

Keywords: RRH; BBU; functional split; quantization; error vector magnitude (EVM).
\end{abstract}

\section{INTRODUCTION}

Mobile data traffic is supposed to increase more than 1000 times from year 2010 to 2020 [1]. Meanwhile, the future 5G system is required to support an increase factor of $10-100$ times of the transmission user data rate and devices with delays as low as a few milliseconds [2]. Operators are facing the challenges to satisfy the explosion of data usage while reducing the cost. A novel mobile network architecture CRAN has been proposed to address these.

In Long Term Evolution (LTE), eNodeB (eNB) contains two main parts: Baseband Unit (BBU) and Remote Radio Head (RRH). RRHs transform the baseband signals from BBUs to radio frequency and then forward it to User Equipments (UEs) by the antennas in the downlink. The process is adverse in the uplink. Meanwhile, BBUs deal with baseband signal processing. RRH is connected to BBU through optical fibers.

$\mathrm{C}-\mathrm{RAN}$ remotes BBUs from RRHs and mitigates them to a BBU pool for centralized processing [3]. The current widely used interface protocol for Inphase $(I)$ and Quadrature $(Q)$ data transmission between RRHs and BBUs is Common Public Radio Interface (CPRI) [4]. The estimated $I Q$ data throughput exceeds $10 \mathrm{Gbps}$ for a 3 sector Base Station (BS) with $20 \mathrm{MHz}$ $4 \times 4$ MIMO. A BBU pool which connects $10-1000 \mathrm{BSs}$ will need vast transmission bandwidth in the fronthaul [5]. This is one of the main issues of C-RAN.

The raw $I Q$ samples transmitted between RRHs and BBUs consume too much bandwidth of the transport network. Thus, an efficient compression scheme is needed for the capacity constrained transport network between RRHs and BBUs. Several solutions have been proposed in [6], such as reducing signal sampling rate, applying non-linear quantization, frequency sub-carrier compression and $I Q$ data compression. Another envisioned method is to change the current functional split architecture between RRH and BBU [7]. This paper investigates on the performance of the last method. Two new functional split architectures are proposed, which move part of the functions in the physical layer of LTE transmission system from BBU to RRH.

For the downlink, the base station is concerned by the transmission side. The baseband signal is defined in a set of discrete symbols, so developing compression scheme is easy. For the uplink, the BS is concerned by the receiver side. As the signal received is affected by noise, it is fundamentally analog. The analog received signal should be quantized before being transmitted from RRHs to BBUs. The quantization has an impact both on the quality of the reception and on the throughput between RRHs and BBUs. Therefore, we focus on LTE uplink.

This paper is organized as follows. In Section II, the Discrete Fourier Transform Spread Orthogonal FrequencyDivision Multiplexing (DFTS-OFDM) transmission system in LTE uplink is briefly introduced. The current and proposed functional split architectures between RRH and BBU are modeled and presented in Section III. The algorithms applied and numerical configurations in the simulation are proposed in Section IV. The simulation results are shown in Section V. At last, this paper is concluded in Section VI.

\section{OVERVIEW OF DFTS OFDM TRANSMISSION SYSTEM}

LTE uplink is based on DFTS-OFDM rather than Orthogonal Frequency-Division Multiplexing Access (OFDMA), which is used for LTE downlink. The Peak to Average Power Ratio (PAPR) of DFTS-OFDM is lower than OFDMA which greatly benefits the User Equipment (UE) in terms of transmit power efficiency and reduced cost of the power amplifier [8]. 


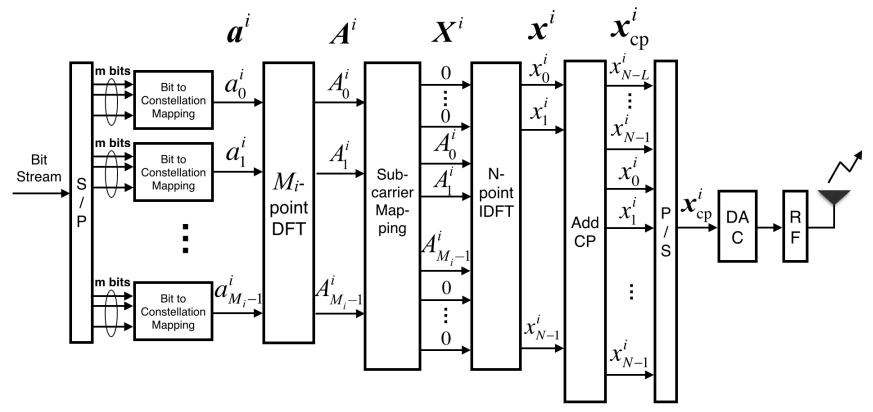

Fig. 1. Localized DFTS-OFDM transmitter.

\section{A. Transmitter}

Let us consider a transmitter $i$ of a DFTS-OFDM system. It converts input bit stream signal to $M_{i}$ modulated sub-carriers over a total of $N$ sub-carriers, as shown in Figure 1. A set of input data bits is firstly collected and then modulated by using QPSK, 16 QAM or 64 QAM, which results in a complex vector $\mathbf{a}^{i}=\left\{a_{0}^{i}, a_{1}^{i}, \ldots, a_{M_{i}-1}^{i}\right\}$. After an $M_{i}$-point unitary Discrete Fourier Transform (DFT), the output $\mathbf{A}^{i}$ is obtained.

Then $\mathbf{A}^{i}$ is mapped to a group of $M_{i}$ adjacent sub-carriers of $\mathbf{X}^{i}$, whose remaining sub-carriers are allocated with zeros. This is called localized sub-carrier mapping. Next, a DFTSOFDM symbol $\mathrm{x}^{i}$ is generated by performing an $N$-point unitary inverse DFT to $\mathbf{X}^{i}$.

To combat multi-path fading, a Cyclic Prefix (CP) is added at the beginning of each symbol $\mathrm{x}^{i}$. A CP is created by copying the last $L$ samples of symbol $\mathbf{x}^{i}$. After a parallel-to-serial operation, $\mathbf{x}_{\mathrm{cp}}^{i}$ is converted to analog signal and modulated to radio frequency, then finally transmitted.

\section{B. White Gaussian Noise Channel Model}

We model the transmission channel with noise between Transmitter $i$ and receiver by adding white Gaussian noise (AWGN). The elements of the noise vector $\mathbf{n}$ follow a normal distribution with mean $\mu=0$ and variance given by

$$
\sigma^{2}=E_{s} \times 10^{-\left(\frac{E_{s}}{N_{0}}\right)}{ }_{d B} / 10
$$

where $E_{s}$ is the average symbol energy defined as:

$$
E_{s}=\mathbb{E}\left(\left|a_{k}^{i}\right|^{2}\right)
$$

and $N_{0}$ is the double-sided power spectral density of the white Gaussian noise.

\section{Receiver}

A block diagram of a DFTS-OFDM receiver for the recovery of signals transmitted from multiple transmitters is illustrated in Figure 2. The signal processing is inverse to that of the aforementioned transmitter. The signal corresponding to Transmitter $j$, denoted by $\mathbf{B}^{j}$, is selected out by the Subcarrier Demapping block. A frequency-domain-equalization (FDE) can be performed after the Sub-carrier Demapping to combat frequency-selective fading and phase distortion. We do not consider equalization in this work.

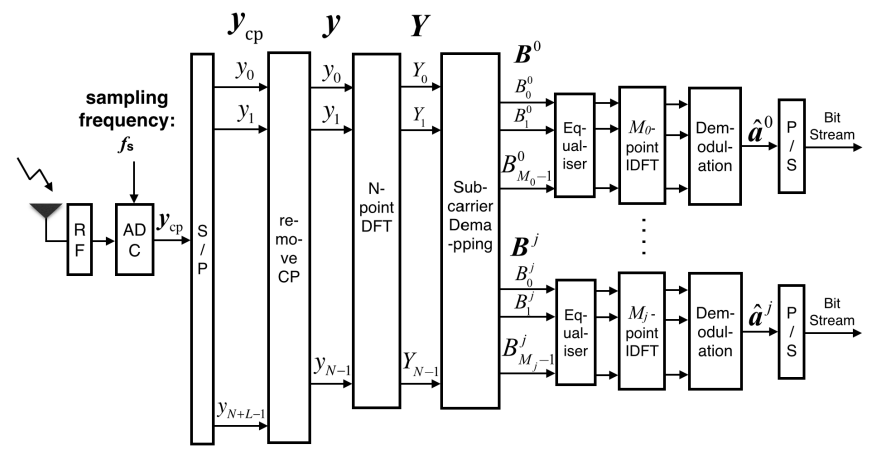

Fig. 2. Localized DFTS-OFDM receiver.

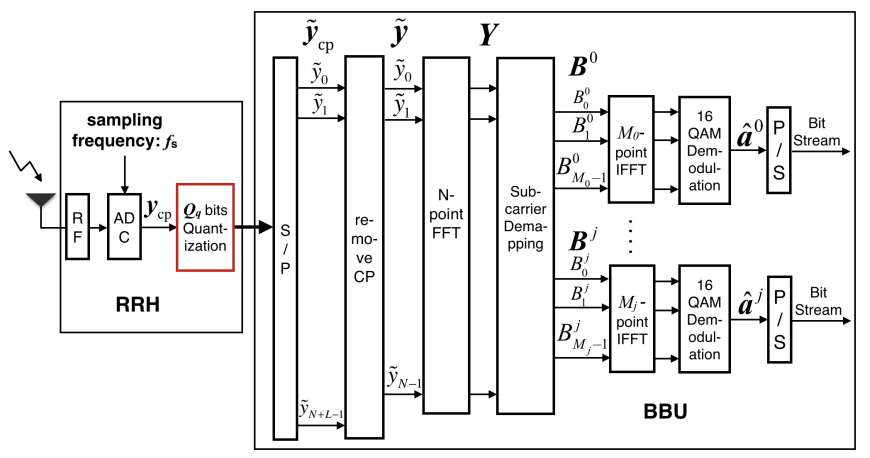

Fig. 3. Method 1 of functional split between RRH and BBU (used in CPRI).

\section{DifFERENT FunCtionAl SPLITS}

\section{A. Current Functional Split Between RRH and BBU}

A model of receiver applying typical functional split is shown in Figure 3, which we denote as Method 1. A $Q_{\mathrm{q}^{-}}$ bits linear quantization is applied to both the real part and the imaginary part of $\mathbf{y}_{\mathrm{cp}}$ separately before it is transmitted from RRH to BBU. The RRH in Method 1 can't identify which and how many Physical Resource Blocks (PRBs) are occupied, thus it has to transmit all the PRBs even if all of them are vacant. Therefore, the transmission rate between RRHs and BBUs is constant, independent of the mobile network load.

\section{B. Proposed Functional Splits Between RRH and BBU}

In LTE transmission, the number of guard sub-carriers is about 40 percent of all the sub-carriers transmitted and the cyclic prefix (CP) also forms 7 to 25 percents of the data transmitted [7]. We consider to remove $\mathrm{CP}$ and the guard sub-carriers before the transmission from RRH to BBU. Two methods of functional split which move "remove CP" and "FFT" blocks from BBU to RRH are proposed. We denote them Method 2 and 3, and detail them hereafter.

1) Method 2 of Functional Split Between RRH and BBU: The model of Method 2 is shown in Figure 4. The difference between Method 1 and 2 is that we move the "remove CP" block and "N-point-FFT" block from BBU to RRH. The new block "PRB usage state detection" detects which sets of 12 sub-carriers corresponding to one PRB, are occupied in $\mathbf{Y}$. 


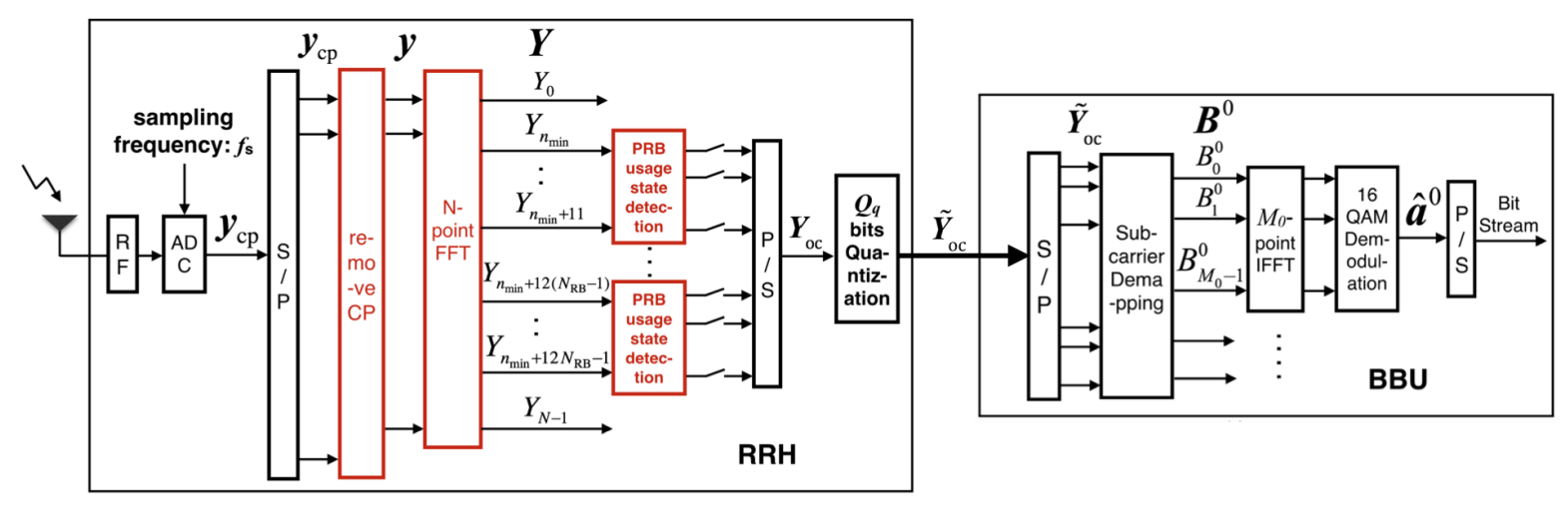

Fig. 4. Method 2 of functional split between RRHs and BBUs.

Usually, not all the PRBs are occupied in LTE transmission. If we can detect which PRBs are occupied or vacant, then we can transmit just the PRBs occupied to further reduce the data transmission rate between RRH and BBU.

One possible method is to calculate the sum of the modulus of 12 elements belonging to a PRB in $\mathbf{Y}$. Assume that $\left\{Y_{12 k+n_{\min }}, Y_{12 k+n_{\min }+1}, \ldots, Y_{12 k+n_{\min }+11}\right\}$ in $\mathbf{Y}$ belong to PRB $k$, where $n_{\min }$ is the index of the first active sub-carrier. The sum of the modulus of the 12 elements is given as

$$
W_{k}=\sum_{n=0}^{11}\left|Y_{12 k+n}\right|^{2}
$$

If $W_{k}$ is less than a threshold, we can decide that the 12 subcarriers in PRB $k$ are vacant.

2) Method 3 of Functional Split Between RRH and BBU:

The model of Method 3 is shown in Figure 5. The dynamic of the signal $\mathbf{y}$ in the frequency domain is higher than $\mathbf{Y}$ in the time domain. In method 2, the signal transmitted from RRH to BBU is in frequency domain. We consider to transform it to time domain before the transmission to reduce the dynamic. To realize this, different from method 2, we perform a 12-point unitary IFFT on $\left\{Y_{12 k+n_{\min }}, Y_{12 k+n_{\min }+1}, \ldots, Y_{12 k+n_{\min }+11}\right\}$ in $\mathbf{Y}$ in Method 3. Then we obtain a vector $\left\{c_{12 k}, c_{12 k+1}, \ldots, c_{12 k+11}\right\}$, where $k=0,1, \ldots, N_{\mathrm{RB}}-1$. The total number of PRBs during one LTE time slot is denoted by $N_{\mathrm{RB}}$. Next, the block "PRB usage state detection" decides if this vector will be quantized and transmitted to BBUs.

In BBUs, the serial "12-point FFT" blocks are used to reverse the 12-point unitary IFFT operation in the RRHs.

\section{Algorithms and Numerical Configurations}

This section present the simulation set up of DFT-Spread OFDM in LTE Uplink physical layer of $20 \mathrm{MHz}$, which includes $N=2048$ sub-carriers where 1200 of them are active. The length of CP $L=160$. Modulation scheme 16 QAM is adapted, thus $m=4$ bits per symbol. "DAC" and "RF" blocks in the transmitter are not considered as well as "ADC" and "RF" in the receiver. Three different methods of functional split between RRH and BBU are applied and the performances will be compared in Section V.

\section{A. Quantization and Frame Arrangement}

In order to minimize the quantization error, a digital automatic gain control is applied. A scaling factor $F_{\mathrm{s}}$ is determined for each block of $N_{\mathrm{s}}$ received $I Q$ samples which is denoted by $\mathbf{z}=\left\{z_{1}, z_{2}, \ldots, z_{N_{\mathrm{s}}-1}\right\}$. Then $\mathbf{z}$ is quantized linearly with $Q_{q}$ bits resolution per complex component based on $F_{\mathrm{s}}$. The scaling factor is sent together with $\mathbf{z}$ from RRHs to BBUs. In order not to introduce a large extra delay to the transmission system, the value of $N_{s}$ should be a factor of $N+L$ for Method 1, and of the total number of sub-carriers occupied during each LTE time slot for Method 2 and 3.

We define the largest absolute value as

$$
V_{\max }=\max _{k=0, \ldots, N_{\mathrm{s}}-1}\left\{\left|\Re\left(z_{k}\right)\right|,\left|\Im\left(z_{k}\right)\right|\right\}
$$

where $\Re\left(z_{k}\right)$ and $\Im\left(z_{k}\right)$ denotes the real and imaginary part of $z_{k}$, respectively.

The corresponding scaling factor is determined as

$$
F_{\mathrm{s}}= \begin{cases}\left\lceil\frac{V_{\max }}{p}\right\rceil & \text { for }\left\lceil\frac{V_{\max }}{p}\right\rceil \leq 2^{Q_{\mathrm{s}}}-1 \\ 2^{Q_{\mathrm{s}}}-1 & \text { for }\left\lceil\frac{V_{\max }}{p}\right\rceil>2^{Q_{\mathrm{s}}}-1\end{cases}
$$

where $p$ is the quantization step for $V_{\max }$ and $Q_{\mathrm{s}}$ is the number of bits used to represent $F_{\mathrm{s}}$.

Then the $I Q$ samples are linearly quantized to $2^{Q_{\mathrm{q}}}$ levels ranging from $-F_{\mathrm{s}}$ to $F_{\mathrm{s}}$. The $n$-th quantization level is given by

$$
q_{n}=-F_{\mathrm{s}} p+\left(n+\frac{1}{2}\right) \cdot \frac{F_{\mathrm{s}} p}{2^{Q_{\mathrm{q}}-1}}
$$

where $n=0,1, \ldots, 2^{Q_{\mathrm{q}}}-1$.

Applying the quantization levels obtained, the $I Q$ samples $z$ are linearly quantized as

$$
\begin{aligned}
& u_{I}(k)=\arg \min _{n}\left|q_{n}-\Re\left(z_{k}\right)\right|, \\
& u_{Q}(k)=\arg \min _{n}\left|q_{n}-\Im\left(z_{k}\right)\right|
\end{aligned}
$$

where $k=0, \ldots, N_{\mathrm{s}}-1$.

Inspired by [9], the $I Q$ samples transmitted from RRH to BBU may be organized as a frame given in Figure 6. The first $Q_{s}$ bits are used to represent $F_{\mathrm{s}}$. Moreover, the references of the corresponding occupied PRBs are also represented by $Q_{\mathrm{r}}$ 


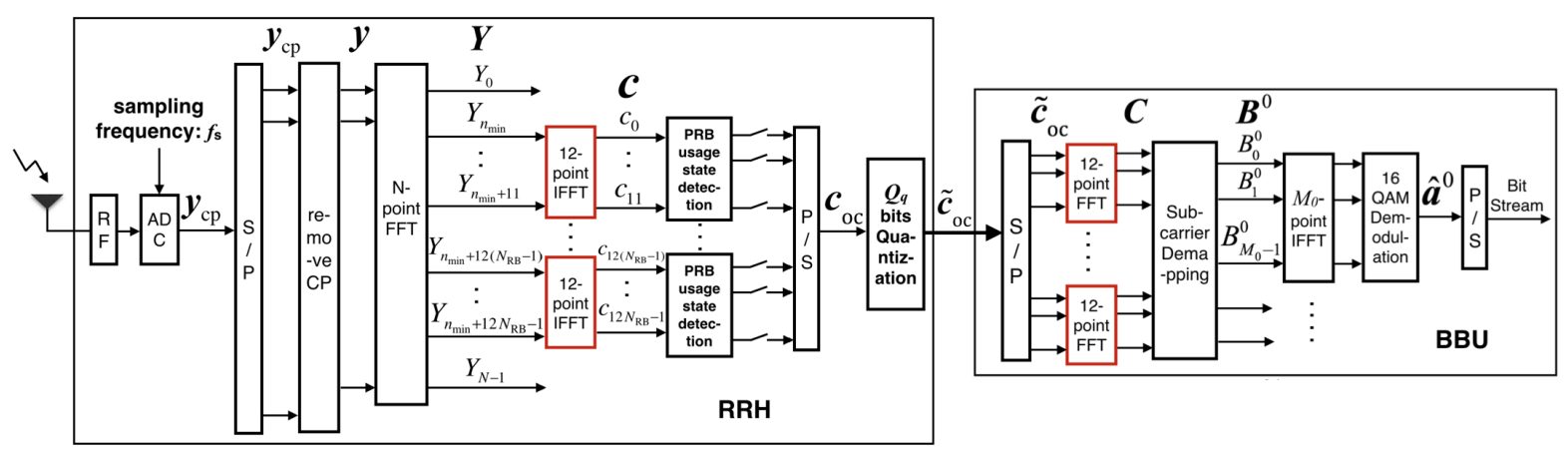

Fig. 5. Method 3 of functional split between RRHs and BBUs.

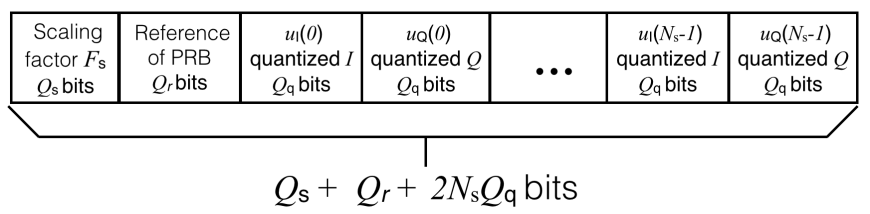

Fig. 6. Possible frame structure.

bits. This is necessary for Method 2 and 3 to identify which PRBs the samples received belong to. For Method $1, Q_{\mathrm{r}}=0$.

Taking into account the transmission of the scaling factor and the references of PRB, the average number of bits used to transport a complex element ( $I$ or $Q$ ) for Method $k$ is:

$$
Q_{\text {eff } k}=\frac{2 N_{\mathrm{s}_{k}} Q_{\mathrm{q}_{k}}+Q_{\mathrm{s}}+Q_{\mathrm{r}}}{2 N_{\mathrm{s}_{k}}}
$$

where $k=1,2,3, N_{\mathrm{s}_{k}}$ is the number of $I Q$ samples in each frame and $Q_{\mathrm{q}_{k}}$ is the number of quantization bits per complex component.

\section{B. Data Transmission Rate From RRH to BBU}

In the following, we will present the formula of data transmission rate calculation from RRH to BBU separately.

1) Data Transmission Rate for Method 1: The data transmission rate between RRH and BBU for Method 1 is given by

$$
D_{1}=F_{\mathrm{os}} \cdot f_{\mathrm{s}} \cdot 2 \cdot Q_{\mathrm{eff} 1} \cdot \frac{1}{R_{\mathrm{c}}}
$$

where $F_{\mathrm{os}}$ is the oversampling factor, $f_{s}$ is the minimum sampling frequency, and $Q_{\mathrm{eff}_{1}}$ is the number of effective quantization bits for each complex element $(I$ or $Q)$ as mentioned in Section IV-A. The factor 2 in Equation 10 results from that both $I$ and $Q$ signals should be transmitted. Due to the Forward Error Correction (FEC) code applied in CPRI, a code rate $R_{\mathrm{c}}$ needs to be considered.

In LTE uplink with $20 \mathrm{MHz}$ bandwidth, there are 2048 subcarriers. Therefore, the minimum sampling frequency $f_{s}=$ $2048 \times \Delta f=2048 \times 15 \mathrm{kHz}=30.72 \mathrm{MHz}$. Oversampling is doing sampling to a signal with a frequency higher than the Nyquist rate (minimum sampling rate). It is applied to improve
TABLE I

SiMULATION PARAMETERS FOR METHOD 1.

\begin{tabular}{|c|c|c|}
\hline PAPAMETER & SYMBOL & VALUE \\
\hline Oversampling factor & $F_{\mathrm{os}}$ & 1 \\
\hline Minimum sampling frequency & $f_{\mathrm{s}}$ & $30.72 \mathrm{MHz}$ \\
\hline Number of samples for each frame & $N_{\mathrm{s}}$ & 138 \\
\hline Number of bits for scaling factor & $Q_{\mathrm{s}}$ & 16 \\
\hline Number of bits for reference of PRB & $Q_{\mathrm{r}}$ & 0 \\
\hline Code rate of FEC applied & $R_{\mathrm{c}}$ & $8 / 10$ \\
\hline
\end{tabular}

the performance of system. The values of the parameters for Method 1 in our simulation are shown in Table I.

2) Data Transmission Rate for Method 2 and 3: The data transmission rate from RRH to BBU for Method 2 is denoted by $D_{2}$, and $D_{3}$ for Method 3. The data transmission rate is given by

$$
D_{k}=N_{\mathrm{Sc}} \cdot \frac{1}{T_{\mathrm{s}}} \cdot 2 \cdot Q_{\mathrm{eff} k} \cdot \frac{1}{R_{\mathrm{c}}} \cdot \eta
$$

where $N_{\mathrm{Sc}}$ is the number of active sub-carriers for data transmission, $T_{\mathrm{s}}$ is the symbol duration, $k=2,3$, and $\eta$ is the assumed PRB utilization ratio. The factors 2 and $R_{\mathrm{c}}$ in Equation 11 are the same as in Equation 10 for Method 1.

TABLE II

SIMULATION PARAMETERS FOR METHOD 2 AND 3.

\begin{tabular}{|c|c|c|}
\hline PAPAMETER & SYMBOL & VALUE \\
\hline Number of active subcarriers & $N_{\mathrm{Sc}}$ & 1200 \\
\hline Symbol duration (for normal CP) & $T_{s}$ & $71.43 \mu \mathrm{s}$ \\
\hline Number of samples for each frame & $N_{\mathrm{s}_{k}}(k=2,3)$ & 12 \\
\hline Number of bits for scaling factor & $Q_{\mathrm{s}}$ & 16 \\
\hline Number of bits for reference of PRB & $Q_{\mathrm{r}}$ & 7 \\
\hline Code rate of FEC applied & $R_{\mathrm{c}}$ & $8 / 10$ \\
\hline Assumed PRB utilization ratio & $\eta$ & $0.5 \mathrm{or} 1$ \\
\hline
\end{tabular}

The value assignments of the parameters in our simulation for method 2 and 3 are shown in Table II.

\section{Error Vector Magnitude}

The error vector magnitude (EVM) is used to quantify the performance of aforementioned different methods of receiver. EVM is defined as

$$
E V M=\sqrt{\frac{\mathbb{E}\left[\left|a_{k}^{i}-\hat{a}_{k}^{i}\right|^{2}\right]}{\mathbb{E}\left[\left|a_{k}^{i}\right|^{2}\right]}}
$$


where $a_{k}^{i}$ are the 16 QAM modulated symbols to be sent in transmitters as shown in Figure 1, and $\hat{a}_{k}^{i}$ are the received symbols before 16 QAM demodulation as shown in Figure 2.

We have also considered the cases with AWGN in the transmission channel between transmitters and receivers.

\section{Simulation Results}

The performances of Method 1, 2 and 3 with and without AWGN have been simulated. Using Equation 10 and 11, we can get the data transmission rate from RRH to BBU for the three different methods and quantization resolution $Q_{\mathrm{q}}$.

\section{A. Performance Comparison Between Method 1 and 2.}
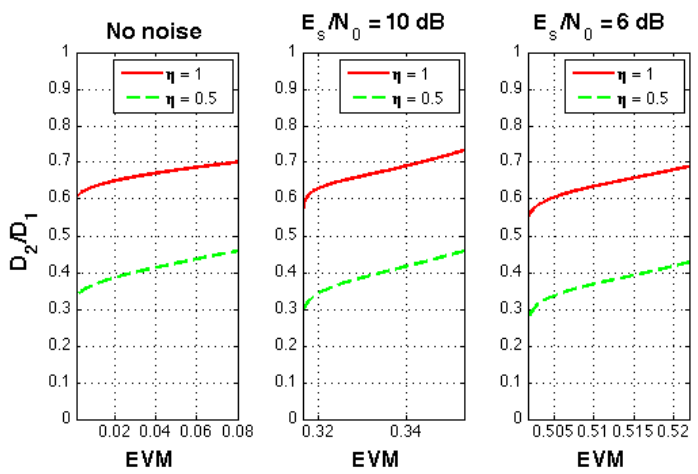

Fig. 7. The relative transmission data rate between Method 1 and 2 with respect to the same value of EVM.

Figure 7 shows the relative transmission data rate between Method 2 and $1\left(\frac{D_{2}}{D_{1}}\right)$ with respect to the same value of EVM. The assumed PRB occupation ratio $\eta=0.5$ and $\eta=1$ are considered in both cases. Scenarios without AWGN, $\frac{E_{\mathrm{s}}}{N_{0}}=$ $10 \mathrm{~dB}$ and $\frac{E_{\mathrm{s}}}{N_{0}}=6 \mathrm{~dB}$ are simulated. It can be observed that, similar results are obtained with adding different levels of white Gaussian noise. Compared with Method 1, Method 2 can save $30 \%$ to $40 \%$ of the bandwidth between RRH and BBU when all the PRBs are occupied, and $55 \%$ to $70 \%$ when only half of the PRBs are occupied.

\section{B. Performance Comparison Between Method 2 and 3.}
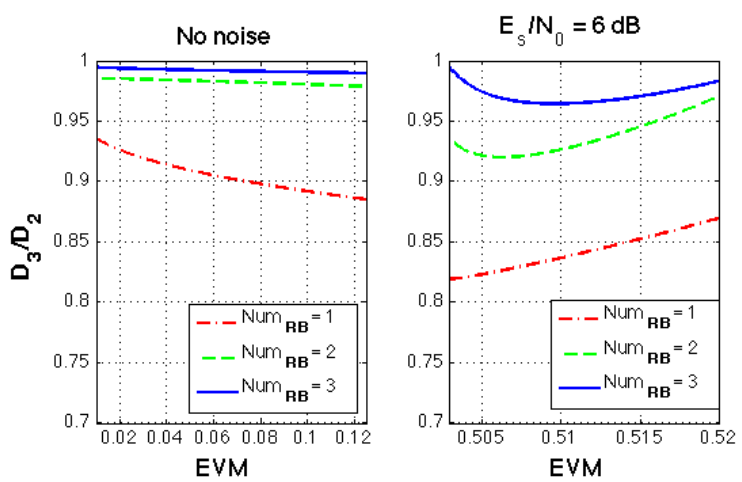

Fig. 8. The relative transmission data rate between Method 2 and 3 with respect to the same value of EVM.
Figure 8 shows the relative transmission data rate between Method 3 and $2\left(\frac{D_{3}}{D_{2}}\right)$ with respect to the same value of EVM. The number of PRBs allocated to each transmitter is denoted by $\mathrm{NUM}_{\mathrm{RB}}$. It can be observed that, compared with Method 2, Method 3 can save $5 \%$ to $10 \%$ of the bandwidth between RRH and BBU when each transmitter is only allocated with one PRB without AWGN, and $12 \%$ to $15 \%$ when $\frac{E_{\mathrm{s}}}{N_{0}}=6 \mathrm{~dB}$. The more PRBs distributed to each transmitter, the less bandwidth gain Method 3 can obtain compared with Method 2. This is because the more PRBs allocated to one transmitter, the more asymmetrical are the serial 12-point IFFT process in the receiver and the $M$-point FFT process in the transmitter.

\section{CONCLUSION}

This paper introduces two new architectures of functional split between RRH and BBU: Method 2 and 3, which have been modeled and simulated. Digital automatic gain control and linear quantization have been applied. In method 2, FFT and resource usage detection are processed in RRH after removing $\mathrm{CP}$. In method 3 , besides the modules in Method 2, a serial 12-point IFFT is made in RRH after FFT and a serial 12-point FFT in BBU. Simulation results illustrate that, Method 2 brings a drop of $30 \%$ to $40 \%$ of the transmission rate between RRHs and BBUs compared with current functional split architecture (Method 1) when all the PRBs are occupied, and up to $70 \%$ when half are occupied. Method 3 can further reduce the transmission rate when UEs are allocated with few PRBs. Applying non-linear quantization algorithms has the potential to further reduce the throughput. Non-linear quantization algorithms are compatible with the different proposed architectures, and will be investigated in a future work.

\section{REFERENCES}

[1] "Enhance mobile networks to deliver 1000 times more capacity by 2020 (white paper)," Nokia Networks.

[2] A. Osseiran, F. Boccardi, V. Braun, K. Kusume, P. Marsch, M. Maternia, O. Queseth, M. Schellmann, H. Schotten, H. Taoka, H. Tullberg, M. Uusitalo, B. Timus, and M. Fallgren, "Scenarios for 5G mobile and wireless communications: the vision of the METIS project," IEEE Communications Magazine, vol. 52, pp. 26-35, May 2014.

[3] C.-L. I, J. Huang, R. Duan, C. Cui, J. Jiang, and L. Li, "Recent Progress on C-RAN Centralization and Cloudification," IEEE Access, vol. 2, pp. 1030-1039, 2014.

[4] "Common Public Radio Interface (CPRI); Interface Specification V6.0," August 2013.

[5] A. Checko, H. Christiansen, Y. Yan, L. Scolari, G. Kardaras, M. Berger, and L. Dittmann, "Cloud RAN for Mobile Networks - A Technology Overview," IEEE Communications Surveys Tutorials, vol. 17, no. 1, pp. 405-426, 2015.

[6] "C-RAN: The Road Towards Green RAN (white paper)," China Mobile Research Institue, Dec 2013.

[7] D. Wubben, P. Rost, J. Bartelt, M. Lalam, V. Savin, M. Gorgoglione, A. Dekorsy, and G. Fettweis, "Benefits and Impact of Cloud Computing on 5G Signal Processing: Flexible centralization through cloud-RAN," IEEE Signal Processing Magazine, vol. 31, pp. 35-44, Nov. 2014.

[8] H. Myung, J. Lim, and D. Goodman, "Single carrier FDMA for uplink wireless transmission," IEEE Vehicular Technology Magazine, vol. 1, pp. 30-38, Sept. 2006.

[9] D. Samardzija, J. Pastalan, M. MacDonald, S. Walker, and R. Valenzuela, "Compressed Transport of Baseband Signals in Radio Access Networks," IEEE Transactions on Wireless Communications, vol. 11, pp. 3216-3225, September 2012. 